\title{
CORRECTION
}

\section{Correction to: The usefulness of full-iterative reconstruction algorithm for the visualization of cystic artery on CT angiography}

Toshihiko Hamamura ${ }^{1}$ - Yoshiko Hayashida ${ }^{1}$ - Yohei Takeshita ${ }^{1} \cdot$ Koichiro Sugimoto $^{1}$ - Issei Ueda ${ }^{1}$. Koichiro Futatsuya ${ }^{1} \cdot$ Shingo Kakeda ${ }^{1} \cdot$ Takatoshi Aoki $^{1} \cdot$ Yukunori Korogi ${ }^{1}$

Published online: 1 April 2020

(C) Japan Radiological Society 2020

\section{Correction to: \\ Japanese Journal of Radiology (2019) 37:526-533 \\ https://doi.org/10.1007/s11604-019-00839-x}

The authors wish to replace the Table 1 of original publication with Table 1 given in this correction.

Publisher's Note Springer Nature remains neutral with regard to jurisdictional claims in published maps and institutional affiliations.

The original article can be found online at https://doi.org/10.1007/ s11604-019-00839-x.

Toshihiko Hamamura

t-hamamura@med.uoeh-u.ac.jp

1 Department of Radiology, University of Occupational and Environmental Health, 1-1 Iseigaoka, Yahatanishi-ku, Kitakyushu 807-8555, Japan 
Table 1 Mean $\Delta$ CT numbers (HU) of main trunk, superficial branch and deep branch of the cystic arteries reconstructed with FBP, AIDR3D, and FIRST from the 30 patients

\begin{tabular}{|c|c|c|c|c|c|c|}
\hline & \multicolumn{2}{|l|}{ Main trunk } & \multicolumn{2}{|c|}{ Superficial branch } & \multicolumn{2}{|l|}{ Deep branch } \\
\hline & $\Delta \mathrm{CT}$ number & HU peak/HU background & $\Delta \mathrm{CT}$ number & HU peak/HU background & $\Delta \mathrm{CT}$ number & HU peak/HU background \\
\hline FBP & $128.52 \pm 56.75$ & $124.49 \pm 55.01 /-4.03 \pm 38.26$ & $88.28 \pm 39.72$ & $94.54 \pm 36.56 / 6.26 \pm 34.44$ & $81.20 \pm 34.85$ & $84.52 \pm 32.56 / 3.33 \pm 28.62$ \\
\hline AIDR3D & $117.21 \pm 56.43$ & $120.26 \pm 55.57 / 3.04 \pm 38.13$ & $78.13 \pm 37.27$ & $84.84 \pm 35.80 / 6.71 \pm 34.49$ & $71.65 \pm 33.23$ & $77.87 \pm 31.98 / 6.22 \pm 29.39$ \\
\hline FIRST & $159.55 \pm 65.83$ & $147.99 \pm 62.07 /-11.55 \pm 36.50$ & $111.64 \pm 48.29$ & $108.51 \pm 41.33 /-3.13 \pm 34.87$ & $100.72 \pm 43.08$ & $100.42 \pm 36.56 /-0.30 \pm 29.18$ \\
\hline P1 & $<0.001$ & & $<0.001$ & & $<0.001$ & \\
\hline $\mathrm{P} 2$ & $<0.001$ & & $<0.001$ & & $<0.001$ & \\
\hline P3 & $<0.001$ & & $<0.001$ & & $<0.001$ & \\
\hline
\end{tabular}

$H U$ Hounsfield unit, FBP filtered back projection, AIDR3D adaptive iterative dose reduction 3D, FIRST forward-projected, model-based, iterative reconstruction solution

$\mathrm{P} 1=$ comparison between the FBP and AIDR 3D images

$\mathrm{P} 2=$ comparison between the FBP and FIRST images

$\mathrm{P} 3=$ comparison between the AIDR 3D and FIRST images 